\title{
Dipole properties of atoms and molecules in the random phase approximation*
}

\author{
Patrick H. S. Martin ${ }^{\dagger}$ \\ Arthur Amos Noyes Laboratory of Chemical Physics, California Institute of Technology, Pasadena, California 91109 \\ W. H. Henneker and Vincent McKoy \\ Centre Européen de Calcul Atomique et Moléculaire, Université de Paris XI, 91-Orsay, France \\ (Received 6 May 1974)

\begin{abstract}
A random phase approximation (RPA) calculation and a direct sum over states is used to calculate second-order optical properties and van der Waals coefficients. A basis set expansion technique is not force the basis functions to satisfy any sum-rule constraints but rather the formalism (RPA) is such that the Thomas-Reiche-Kuhn sum rule is satisfied exactly. Central attention is paid to the dynamic polarizability from which most of the other properties are derived. Application is made to helium and molecular hydrogen. In addition to the polarizability and van der Waals coefficients, results are given for the molecular anisotropy of $\mathrm{H}_{2}$, Rayleigh scattering cross sections, and Verdet constants as a function of frequency. Agreement with experiment and other theories is good. Other energy weighted sum rules are calculated and compare very well with previous estimates. The practicality of our method suggests its applications to larger molecular systems and other properties.
\end{abstract} \\ used and no continuumlike functions are included in the basis. However, unlike other methods we do
}

\section{INTRODUCTION}

Atomic and molecular polarizabilities (along with the anisotropy in the case of molecules) play a central role in the investigation of such important properties as the optical refractivity, ${ }^{1}$ Faraday rotation, ${ }^{2}$ Rayleigh scattering cross sections, ${ }^{3}$ and van der Waals coefficients. ${ }^{4}$

Experimental data on these atomic and molecular properties are far from satisfactory over a wide range of frequency values (e.g., in the vacuum uv). Experimental data are especially scarce in the case of Rayleigh scattering cross sections and depolarization ratios as well as in the rotational and vibrational Raman scattering cross section where the polarizability tensor and its variation with internuclear distance are required. ${ }^{5} \mathrm{Al}-$ though Raman scattering experiments could in principle provide a direct measurement of the polarizability anisotropy, very few values have actually been obtained.

Theoretical predictions of atomic and molecular polarizabilities are therefore useful in the analysis of available experimental data and are frequently the only available estimates of many second-order optical properties. For this reason considerable effort has been put on the theoretical and semiempirical computation of the dynamic polarizability of atomic and molecular systems. These methods involve procedures which circumvent the infinite sum over intermediate states in the Kramers-Heisenberg dispersion formula. One such class of methods consists of employing oscillator strength distributions and excitation energies from theoretical calculations or experiment to construct bounds for the polarizability using the theory of moments, ${ }^{6}$ Padé approximants, ${ }^{7}$ Gaussian quadratures, ${ }^{8}$ and similar bounding techniques. ${ }^{9}$

An alternative approach is the semiempirical one ${ }^{10}$ in which oscillator strength distributions from experiment are used in the Kramers-Heisenberg dispersion formula. In cases where sufficient spectral data are available, accurate results can be obtained.

Another alternative, and this one more closely related to ours, is the variational one. ${ }^{11-16}$ In this method the oscillator strength distributions are determined from a variational procedure subject to certain sumrule constraints. The resulting finite spectrum does not necessarily represent an actual one (i.e., not all the poles correspond to actual states of the system) but can provide accurate values of the polarizability.

The method used in the present work is a direct sum over states procedure in which the spectrum is obtained from the random phase approximation. Next we describe our approach and apply it to several second-order optical properties of helium and molecular hydrogen. In general, agreement with previous computations and with

TABLE I. Frequency-dependent polarizability for helium.

\begin{tabular}{lcr}
\hline$\omega\left(\mathrm{a}, \mathrm{u}_{.}\right)^{\mathrm{a}}$ & This work & \multicolumn{1}{c}{$\mathrm{AG}^{\bullet}$} \\
\hline 0.000 & 1.322 & 1.32219 \\
0.100 & 1.336 & 1.33622 \\
0.200 & 1.380 & 1.38056 \\
0.300 & 1.462 & 1.46291 \\
0.400 & 1.600 & 1.60071 \\
0.500 & 1.834 & 1.83418 \\
0.600 & 2.275 & 2.27585 \\
0.700 & 3.435 & 3.43453 \\
0.750 & 5.487 & 5.48586 \\
$0.24 \pi^{b}$ & 5.832 & 5.83165 \\
0.790 & 25.23 & 25.238 \\
$0.252 \pi^{c}$ & 32.45 & 32.459 \\
0.800 & -49.57 & -49.532 \\
0.805 & -16.85 & -16.842 \\
\hline \hline
\end{tabular}

\%o convert from atomic units to angstroms use $\lambda(\AA) \simeq 455.6 / \omega$ (a. u. ).

b. $0.24 \pi 0.7540$.

${ }^{c} 0.252 \pi \sim 0.7917$.

d(12S/8P) basis. See text for details.

See Ref. 26 of text. 
TABLE II. Frequency dependent polarizability for helium $\alpha(\omega)$ (a. u.)-RPA-results in different bases. ${ }^{2}$

\begin{tabular}{lccc}
\hline \hline$\omega{\text { (a.u. })^{\mathrm{b}}}^{\mathrm{b}}$ & $(11 S / 5 P)$ & $(12 S / 8 P)$ & $\mathrm{AG}^{\mathrm{c}}$ \\
\hline 0.000 & 1.274 & 1.322 & 1.32219 \\
0.100 & 1.288 & 1.336 & 1.33622 \\
0.200 & 1.331 & 1.380 & 1.38056 \\
0.300 & 1.411 & 1.462 & 1.46291 \\
0.400 & 1.546 & 1.600 & 1.60071 \\
0.500 & 1.773 & 1.834 & 1.83418 \\
0.600 & 2.204 & 2.275 & 2.27585 \\
0.700 & 3.333 & 3.435 & 3.43453 \\
0.750 & 5.323 & 5.487 & 5.48586 \\
$0.24 \pi$ & 5.656 & 5.832 & 5.83165 \\
0.790 & 23.74 & 25.23 & 25.238 \\
$0.252 \pi$ & 30.15 & 32.45 & 32.459 \\
0.800 & -54.18 & -49.57 & -49.532 \\
0.805 & -17.26 & -16.85 & -16.842 \\
\hline \hline
\end{tabular}

asee text for description of basis set.

To convert from atomic units to angstroms, use $(\AA) \simeq 455.6$ / $\omega$ (a.u.).

'Reference 26.

$\mathrm{d} 0.24 \pi \sim 0.7540$.

$\bullet 0.252 \pi \sim 0.7917$.

the available experimental data is seen to be good. The basis sets and excitation spectra used are given in Appendices $\mathrm{B}$ and $\mathrm{C}$, respectively.

\section{THEORY}

Application of the semiclassical theory of the interaction of radiation with matter is known to lead to the correct results for a wide variety of phenomena of interest. Such is the case, for example, of the dynamic dipole polarizability of atomic and molecular systems, for

TABLE III. Frequency-dependent polarizability of helium. Comparison with other results.

\begin{tabular}{llll}
\hline \multicolumn{3}{l}{ Comparison with other results. } \\
\hline \hline$(\AA)$ & Experimental $^{\mathrm{a}}$ & This work & \\
\hline 20587.0 & 1.3868 & 1.3225 & PWL $^{\mathrm{b}}$ \\
15300.0 & 1.3873 & 1.3228 & $\ldots$ \\
14756.0 & 1.3876 & 1.3229 & $\ldots$ \\
10142.0 & 1.3892 & 1.3244 & $\ldots$ \\
9227.0 & 1.3899 & 1.3249 & $\ldots$ \\
8266.8 & 1.3908 & 1.3258 & 1.388 \\
7247.2 & 1.3923 & 1.3271 & 1.389 \\
5462.2 & 1.3971 & 1.3313 & 1.390 \\
4801.3 & 1.4004 & 1.3342 & 1.395 \\
4359.0 & 1.4009 & 1.3369 & 1.398 \\
4047.0 & 1.4037 & 1.3394 & 1.401 \\
3907.0 & 1.4052 & 1.3407 & 1.404 \\
3664.0 & 1.4082 & 1.3434 & $\ldots$ \\
3342.0 & 1.4133 & 1.3479 & 1.408 \\
3132.0 & 1.4175 & 1.3517 & $\ldots$ \\
3022.0 & 1.4200 & 1.3540 & 1.417 \\
3968.0 & 1.4215 & 1.3552 & $\ldots$ \\
2926.0 & 1.4226 & 1.3562 & $\ldots$ \\
2894.0 & 1.4236 & 1.3570 & $\ldots$ \\
2753.0 & 1.4279 & 1.3609 & 1.423 \\
\hline \hline
\end{tabular}

Experimental refractivity data from: C. R. Mansfield and E. R. Peck, J. Opt. Soc. Am. 59, 199 (1969); C. Cuthbertson and M. Cuthbertson, Proc. R. Soc. Lond. 135, 40 (1932). ${ }^{b}(12 S / 8 P)$ basis. See text for details.

'See Ref. 6 of text.

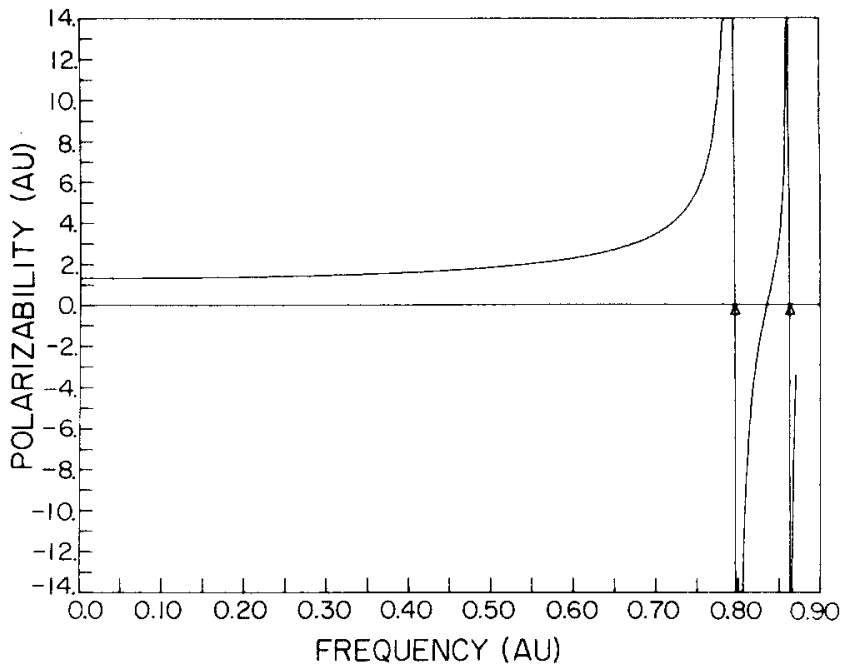

FIG. 1. Dispersion of the dipole polarizability of He for frequencies up to the second pole.

which the formal Kramers-Heisenberg dispersion formula applies, ${ }^{17}$

$$
\boldsymbol{\alpha}(\omega)=\mathbf{S} \frac{f_{n o}}{\omega_{n o}^{2}-\omega^{2}}
$$

where the $\mathbf{S}_{n \neq 0}$ is a summation over the discrete part and an integration over the continuous part of the spectrum. Atomic units are used throughout unless otherwise stated. The $f_{n o}$ and $\omega_{n o}$ are the oscillator strength and the excitation energy, respectively, for the transition between state $|n\rangle$ and the ground state $|0\rangle$ of the system.

Similarly, the long range van der Waals force coefficients for the interaction between two species $A$ and $B$ is given by ${ }^{18,19}$

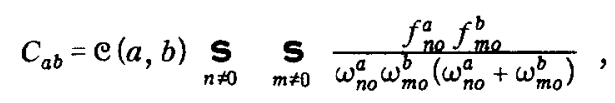

where $\mathcal{C}(a, b)$ is a constant which depends on the nature (whether atom or molecule) of the species and symmetry of the component of the polarizability to which the $C_{a b}$ in question is related. Appendix $A$ gives the values of e $(a, b)$ for various atom-molecule and molecule-molecule interactions. These may be easily derived from the definitions of the van der Waals coefficients in terms of the polarizability for imaginary frequencies as given elsewhere. ${ }^{10}$

We assume the following definitions for the oscillator strengths and polarizability components:

$$
\begin{aligned}
& f_{n o}^{\prime \prime}=\frac{2}{3} \omega_{n o}\left|\left\langle 0\left|\sum_{i=1}^{N} z_{i}\right| n\right\rangle\right|^{2}, \\
& f_{n o}^{1}=\frac{4}{3} \omega_{n o}\left|\left\langle 0\left|\sum_{i=1}^{N} x_{i}\right| n\right\rangle\right|^{2}, \\
& \alpha_{g \varepsilon}(\omega)=\alpha_{11}(\omega)=3 \underset{n \neq 0}{\mathbf{s}} \frac{f_{n e}^{\prime \prime}}{\omega_{n o}^{2}-\omega^{2}},
\end{aligned}
$$




$$
\alpha_{x x}(\omega)=\alpha_{1}(\omega)=\frac{3}{2} \underset{m \neq 0}{s} \frac{f_{n o}^{\perp}}{\omega_{m o}^{2}-\omega^{2}}
$$

In the above equations $z$ points along the internuclear axis. The trace of the polarizability is given by

$$
\alpha(\omega) \frac{1}{3}\left[2 \alpha_{\perp}(\omega)+\alpha_{11}(\omega)\right]
$$

and the anisotropy by

$$
\gamma(\omega)=\alpha_{11}(\omega)-\alpha_{1}(\omega) .
$$

Several macroscopic properties are directly related to and determined by the microscopic atomic and molecular polarizability and anisotropy. The optical refractivity is connected to $\alpha(\omega)$ by the Lorenz-Lorentz formula,

$$
\eta(\omega)-1=2 \pi N_{0} \alpha(\omega)
$$

where $N_{0}$ is the number density of a dilute gas. The Verdet constant is related to the frequency derivative of the polarizability through the Becquerel formula

$$
V(\omega)=\left(2 c^{2}\right)^{-1} \omega[\operatorname{dn}(\omega) / d \omega],
$$

where $c$ is the velocity of light. The Rayleigh scattering cross section involves both the trace of the polarizability $\alpha(\omega)$ and the anisotropy $\gamma(\omega)$,

$$
\sigma(\omega)=\left(8 \pi / 9 c^{4}\right) \omega^{4}\left[3 \alpha(\omega)^{2}+\frac{2}{3} \gamma(\omega)^{2}\right] ;
$$

$\alpha(\omega)$ and $\gamma(\omega)$ also completely determine the Rayleigh depolarization ratios and Raman scattering cross sections. Vibrational Raman scattering is analogously determined by the variation of the polarizability components with internuclear distance. Thus a detailed knowledge of the dipole polarizability of system conveys a wealth of information on its optical properties.

The difficulty with Eqs. (1) and (2) is that they assume a complete knowledge of the excitation spectrum of the system involved, and the determination of all the excitation energies and oscillator strengths in intractable. The cumbersome infinite summations over the whole spectrum in Eqs. (1) and (2) are typical of the results

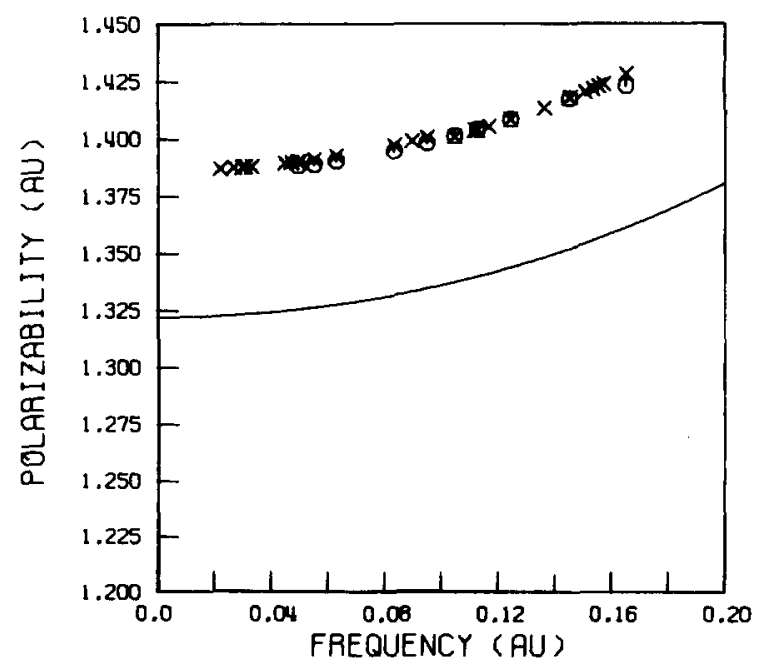

FIG. 2. Frequency-dependent polarizability of He. Comparison of this work with experiment $(x)$ and results from Ref. 6 (o).
TABLE IV. Verdet constant for helium-RPA. Comparison of different bases.

\begin{tabular}{lcc}
\hline \hline$\lambda(\AA)$ & $\begin{array}{l}V(\omega)^{\mathbf{a}} \\
(11 S / 5 P)^{\mathrm{b}}\end{array}$ & $\begin{array}{l}V(\omega)^{\mathbf{a}} \\
(12 S / 8 P)^{\mathrm{b}}\end{array}$ \\
\hline 9875 & 0.1459 & 0.1497 \\
9000 & 0.1759 & 0.1804 \\
8500 & 0.1974 & 0.2025 \\
8000 & 0.2230 & 0.2288 \\
7500 & 0.2540 & 0.2606 \\
7000 & 0.2920 & 0.2995 \\
6500 & 0.3392 & 0.3480 \\
6000 & 0.3990 & 0.4093 \\
5780 & 0.4304 & 0.4415 \\
5500 & 0.4761 & 0.4884 \\
5460 & 0.4833 & 0.4957 \\
5000 & 0.5782 & 0.5932 \\
4500 & 0.7174 & 0.7359 \\
4360 & 0.7655 & 0.7853 \\
4000 & 0.9143 & 0.9379 \\
3635 & 1.115 & 1.144 \\
2500 & 2.467 & 2.530 \\
$1215.7^{\mathrm{c}}$ & 14.25 & 14.61 \\
1000 & 26.56 & 27.24 \\
\hline \hline
\end{tabular}

In units of microminutes. Oersted ${ }^{-1} \cdot \mathrm{cm}^{-1}$ (at $0^{\circ} \mathrm{C}$ and 1 atm). 'See text for description of basis sets. The results for the $(10 S / 13 P)$ basis are identical to the $(12 S / 8 P)$ to four significant figures.

${ }^{c}$ Lyman alpha radiation.

of second-order perturbation theory. The method we employ to circumvent these summations is to replace the true spectrum by a finite number of excitation frequencies and oscillator strengths as obtained from the equations of motion (EOM) ${ }^{20-22}$ method. The over-all procedure is a direct sum over states method analogous to that of Refs. 11 and 12 (and references therein). The major difference, however, is the procedure for obtaining the oscillator strength distribution. A characteristic feature of our approach is that no constraints are put on the basis functions used in our calculations. Instead, ordinary Gaussian basis sets are used in the HartreeFock (HF) ground state calculation, and the HF orbitals provide the particle-hole basis needed for our calculation. Next we outline the EOM theory which has been fully discussed elsewhere. ${ }^{20,21}$

The EOM method is a many-body approach to the calculation of excitation energies and oscillator strengths in which these quantities are calculated directly without requiring elaborate wavefunctions for the states involved. It is specifically designed for calculation of relative quantities rather than absolute energies and total wavefunctions. In the EOM method an operator $\mathrm{O}_{\lambda}^{+}$is defined such that

$$
O_{\lambda}^{+}|0\rangle=|\lambda\rangle
$$

where $|0\rangle$ is the ground state and $|\lambda\rangle$ is some excited state. $\mathrm{O}_{\lambda}^{+}$is then shown to satisfy the following equation of motion:

$$
\left\langle 0\left|\left[\delta O_{\lambda}, H, O_{\lambda}^{+}\right]\right| 0\right\rangle=\omega_{\lambda}\left\langle 0 \mid\left[\delta O_{\lambda}, O_{\lambda}^{+} \mid\right] 0\right\rangle,
$$

where $\omega_{\lambda}$ is the transition frequency and $\delta O_{\lambda}^{+}$is a varia- 
tion in the amplitudes specifying $O_{\lambda}^{+}$. The double commutator is defined as

$$
2[A, B, C]=[[A, B], C]+[A,[B, C]]
$$

for any three operators $A, B$, and $C$.

If $O_{\lambda}^{+}$is limited to single particle-hole components $(1 p-1 h)$, i.e.,

$$
O_{\lambda}^{+}(S M)=\sum_{m \gamma}\left[Y_{m \gamma}(\lambda) C_{m \gamma}^{+}(S M)-Z_{m \gamma}(\lambda) C_{m \gamma}(\overline{S M})\right],
$$

where $C_{m r}^{+}(S M)$ and $C_{m \gamma}(\overrightarrow{S M})$ are spin adapted particlehole creation and annihilation operators. The amplitudes $\mathbf{Y}(\lambda)$ and $Z(\lambda)$ satisfy the equation

$$
\begin{gathered}
\left(\begin{array}{cc}
\mathbf{A} & \mathbf{B} \\
-\mathbf{B}^{*} & -\mathbf{A}^{*}
\end{array}\right)\left(\begin{array}{l}
\mathrm{Y}(\lambda) \\
\mathrm{Z}(\lambda)
\end{array}\right)= \\
=\omega_{\lambda}\left(\begin{array}{ll}
\mathrm{D} & 0 \\
0 & \mathrm{D}
\end{array}\right)\left(\begin{array}{l}
\mathbf{Y}(\lambda) \\
\mathbf{Z}(\lambda)
\end{array}\right) .
\end{gathered}
$$

The form of the matrices A, B, and D can be found in Refs. 20 and 21. If the Hartree-Fock approximation is used for the ground state, $D$ becomes the unit matrix and the RPA equations follow :

$$
\left(\begin{array}{rr}
\mathbf{A} & \mathbf{B} \\
-\mathbf{B}^{*} & -\mathbf{A}^{*}
\end{array}\right)\left(\begin{array}{l}
\mathrm{Y}(\lambda) \\
\mathbf{Z}(\lambda)
\end{array}\right)=\omega_{\lambda}\left(\begin{array}{l}
\mathrm{Y}(\lambda) \\
\mathbf{Z}(\lambda)
\end{array}\right) .
$$

\begin{tabular}{|c|c|c|c|c|c|}
\hline$\lambda(\AA ̊)$ & $\begin{array}{l}\text { Experi- } \\
\text { mental }^{\mathrm{a}}\end{array}$ & $\begin{array}{l}\text { Semiem- } \\
\text { pirical }^{b}\end{array}$ & $\mathrm{KCH}^{\circ}$ & $\begin{array}{l}\text { This } \\
\text { work }^{\text {d }}\end{array}$ & PWL" \\
\hline 9875 & $\ldots$ & 0.161 & 0.1499 & 0.1497 & $\cdots$ \\
\hline 9000 & 0.205 & 0.194 & 0.1807 & 0.1804 & 00 \\
\hline 8500 & 0.221 & 0.218 & 0.2027 & 0.2025 & 0.224 \\
\hline 8000 & 0.246 & 0.246 & 0.2290 & 0.2288 & 0.253 \\
\hline 7500 & 0.283 & 0.281 & 0.2609 & 0.2606 & 0.288 \\
\hline 7000 & 0.325 & 0.323 & 0.2999 & 0.2995 & 0.332 \\
\hline 6500 & 0.376 & 0.375 & 0.3484 & 0.3480 & 0.385 \\
\hline 6000 & 0.441 & 0.441 & 0.4098 & 0.4093 & 0.453 \\
\hline 5780 & 0.474 & 0.476 & 0.4421 & 0.4415 & $\cdots$ \\
\hline 5500 & 0.523 & 0.526 & 0.4890 & 0.4884 & 0.540 \\
\hline 5460 & 0.531 & 0.534 & 0.4963 & 0.4957 & $\cdots$ \\
\hline $5 \times 0$ & 0.638 & 0.639 & 0.5939 & 0.5931 & 0.657 \\
\hline $45 \cup 0$ & 0.800 & 0.793 & 0.7368 & 0.7359 & 0.815 \\
\hline 4360 & 0.854 & 0.846 & 0.7862 & 0.7853 & $\cdots$ \\
\hline 4000 & 1.011 & 1.01 & 0.9390 & 0.9379 & 1.039 \\
\hline 3635 & 1.249 & 1.23 & 1.145 & 1.144 & $\cdots$ \\
\hline 2500 & ... & 2.73 & 2.533 & 2.530 & $\cdots$ \\
\hline $1215.7^{f}$ & $\cdots$ & 15.5 & 14.28 & 14.61 & $\cdots$ \\
\hline 1000 & $\cdots$ & 27.5 & 25.28 & 27.24 & $\cdots$ \\
\hline
\end{tabular}

Higher order solutions of the EOM equations can be derived and have been used to calculate the spectra of

TABLE V. Verdet constants for helium-RPA. Comparison with other work.

asee Ref. 29 of text.

${ }^{b}$ A. Dalgarno and A. E. Kingston, Proc. R. Soc. A 259, 424 (1960).

'See Ref. 27 of text.

${ }^{d}$ Units of microminutes. Oersteds ${ }^{-1} \cdot \mathrm{cm}^{-1}$ (at $0^{\circ} \mathrm{C}$ and $1 \mathrm{~atm}$ ).

See Ref. 6 of text.

${ }^{i}$ Lyman $\alpha$ radiation.

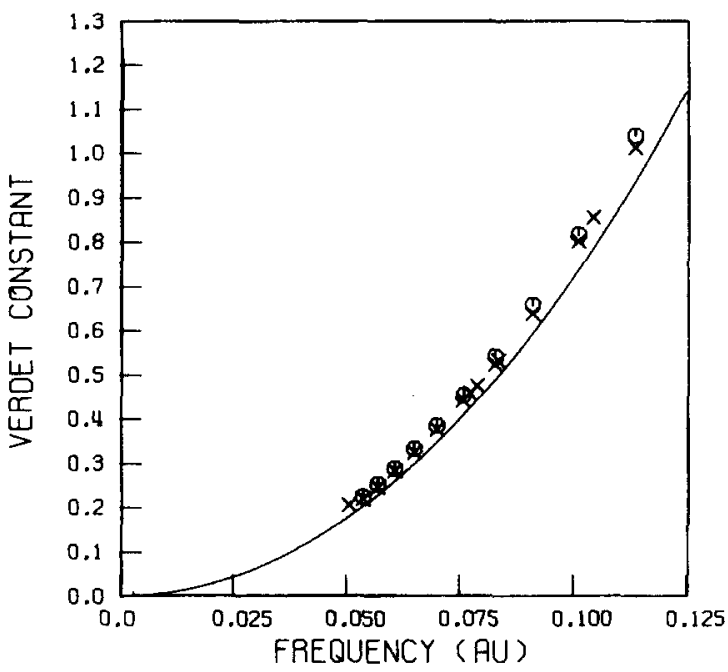

FIG. 3. Verdet constants of He (in units of microminutes. Oersted ${ }^{-1} \cdot \mathrm{cm}^{-1}$ at $0^{\circ} \mathrm{C}$ and $\left.1 \mathrm{~atm}\right)$. Comparison of this work with experiment $(x)$ and results of Ref, $6(0)$.

several molecular systems with excellent agreement with experiment as well as with other theoretical results. ${ }^{22}$ In this work however all the results are computed to the RPA level only.

In the context of the present work, the RPA has the important property of satisfying the Thomas-ReicheKuhn sum rule. This has been formally shown to be the case to the extent of completeness of the basis used in the calculation. ${ }^{23}$ Actual computations have shown that reasonably sized bases provide accurate values of the $S(0)$ sum rule (see below). As a consequence of this fact, no special sum rule constraints have to be put on the basis functions. We have also found that the other sum rules are approximately satisfied. The solution of the RPA equations in a discrete basis leads to a discrete set of oscillator strengths which are used directly to estimate $\alpha(\omega)$, Eq. (1). Some of these $f_{n o}$ 's and $\omega_{n o}$ 's represent approximations to actual excited states of the system but the discrete virtual states do not correspond to actual transitions. Our expression for the polarizability is thus

$$
\alpha(\omega)=\sum_{n=1}^{M} \frac{f_{n o}}{\omega_{n a}^{2}-\omega^{2}},
$$

where $M$ is the number of states considered (the number of excitations).

The fact that there is no need to go to higher-order approximation beyond the RPA to obtain good results for $\alpha(\omega)$ and related properties, and that the equations can be solved without using any continuumlike functions, makes the present approach extremely applicable to larger molecular systems and other properties.

\section{RESULTS AND DISCUSSION}

Helium was calculated in three different contracted Gaussian basis sets : $(11 S / 5 p),(12 S / 8 P)$, and $(10 S / 13 P)$ with exponents suggested by Huzinaga ${ }^{24}$ and additional diffuse $S$ functions and valencelike $P$ functions. ${ }^{25}$ Additional details of the basis sets used will be found in Ap- 
pendix $B$. We compare the results in the three bases and show that while there is a considerable change going from $(11 S / 5 P)$ to $(12 S / 8 P)$ little is gained in going to the larger basis. In these calculations, 5,8 , and 12 poles of $p$ symmetry are obtained. The corresponding oscillator strengths and transition energies are found in Appendix C.

For molecular hydrogen we used an uncontracted $(8 S / 5 P)$ Gaussian basis set with exponents suggested by Huzinaga. ${ }^{24}$ See Appendix $B$ for further basis set information. From this calculation we have obtained 7 poles of ${ }^{1} \Pi_{u}$ symmetry and 14 poles of ${ }^{1} \Sigma_{u}^{+}$symmetry. The resulting excitation spectrum is listed in Appendix $C$. The calculation was done at a fixed internuclear distance of $R=1.4 \mathrm{a}$.u. No vibrational averaging is done. Further comments on the effect of this averaging on the calculated properties will be given in the following.

\section{A. Dynamic dipole properties}

\section{Helium}

In Table I we compare our results for the frequencydependent polarizability with the exact numerical solution of the time-dependent Hartree-Fock equations (to which the RPA is equivalent) given by Alexander and Gordon. ${ }^{26}$ Agreement is excellent. The importance of this comparison is that we are able to obtain essentially exact solutions of the RPA equations using only ordinary Gaussian basis sets. This is particularly important in the present case since we are computing a quantity such as the dipole polarizability which formally involves the continuum (through the Kramers-Heisenberg formula)

TABLE VI. Rayleigh scattering cross sections for heliumRPA. Comparison of different bases.

\begin{tabular}{lll}
\hline \hline$\lambda(\AA) \mathbf{e}$ & $\begin{array}{l}\sigma(\omega)^{\mathrm{b}} \\
(11 S / 5 P)^{\mathbf{c}}\end{array}$ & $\begin{array}{l}\sigma(\omega)^{\mathrm{b}} \\
(8 S / 13 P)^{\mathbf{c}}\end{array}$ \\
\hline$\infty$ & 0.0 & 0.0 \\
20586.0 & 0.002593 & 0.002789 \\
15300.0 & 0.008507 & 0.009151 \\
14756.0 & 0.009833 & 0.01058 \\
10142.0 & 0.04416 & 0.04750 \\
9227.0 & 0.06453 & 0.06941 \\
8266.8 & 0.1003 & 0.1079 \\
7247.2 & 0.1701 & 0.1830 \\
5462.2 & 0.5305 & 0.5706 \\
4801.3 & 0.8926 & 0.9600 \\
4359.0 & 1.319 & 1.419 \\
4047.0 & 1.782 & 1.917 \\
3907.0 & 2.056 & 2.211 \\
3664.0 & 2.669 & 2.870 \\
3342.0 & 3.882 & 4.174 \\
3132.0 & 5.061 & 5.442 \\
3022.0 & 5.859 & 6.300 \\
2968.0 & 6.309 & 6.783 \\
2926.0 & 6.689 & 7.192 \\
2894.0 & 6.998 & 7.524 \\
2753.0 & 8.595 & 9.240 \\
\hline \hline
\end{tabular}

'Wavelengths at which experimental refractivity data are available.

In units of $10^{-28} \mathrm{~cm}^{2}$.

'See text for description of basis sets. The (10S/13P) results are identical to the $(12 S / 8 P)$ values to four significant digits.

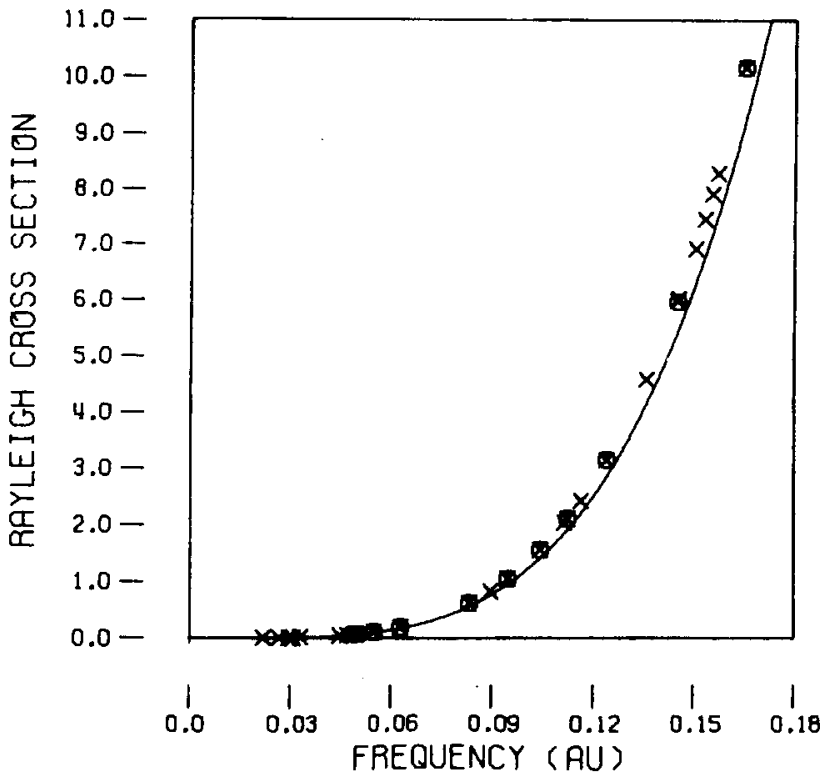

FIG. 4. Rayleigh scattering cross sections of He (in units of $10^{-28} \mathrm{~cm}^{2}$ ). Comparison of this work with experiment and results of Ref. 6 .

but using only square-integrable functions. This is a highly desirable feature of a method that is to be extended to molecular calculations.

In Table II we show the results we obtained in the continuum (through the Kramers-Heisenberg formula) three different basis sets used. $\alpha(\omega)$ was calculated at frequencies for which the exact numerical results cited above are available. The $P$ basis used in the $(12 S / 8 P)$ calculation is fairly "complete" in the sense that it provides values of $\alpha(\omega)$ which do not change appreciably in going to the larger $13 P$ basis. For this reason the results of the larger basis are not shown in the table. Table III shows the $(12 S / 8 P)$ results together with the experimental values (calculated from refractivity measurements through the Lorenz-Lorentz formula) and the moment theory bounds results of Ref.6. Agreement of our values with experiment is good, the difference being less than $5 \%$ in all cases. Moreover this difference is reasonably constant over the range of frequencies for which experimental data are available. This suggests that if our results are scaled so that our static value agrees with the static polarizability extrapolated from the experiments, close agreement can be obtained for all other frequencies. This situation is exactly analogous to what was found by Hurst et al. ${ }^{27}$

Figure 1 shows the dispersion curve of $\alpha(\omega)$ of helium from zero frequency out to the second pole. The poles are marked on the axis by small triangles. The values given by this curve agree with those of Alexander and Gordon $^{26}$ even for frequencies above the first pole.

Figure 2 shows a smaller section (in an expanded scale) of the dispersion curve in the region for which experiments and other calculated results are available. Our curve parallels closely the experimental points. From this it is reasonable to expect accurate values for the frequency derivative, $d \alpha(\omega) / d \omega$, required to predict 
Verdet constants. Here we use the Becquerel formula [Eq. (10)] which is believed to hold for atoms with reasonable accuracy. The modified Becquerel formula which is more widely used contains a empirical (frequency-independent) factor.

Table IV shows the values we obtain for the Verdet constant in the different basis sets. Again it is clear that the intermediate size basis was enough to describe properly the property at hand. Table V shows a comparison of our $(12 S / 8 P)$ results with experimental and semiempirical data as well as with other theoretical calculations. Agreement of our values with both experimental and semiempirical data is very good, especially since Ingersoll and Liebenberg ${ }^{28}$ found that helium conforms to the Becquerel formula to within $4 \%$ only (i.e., they find 0.96 for the average value of the constant involved in the modified Becquerel formula). Our values are also in excellent agreement with a previous timedependent coupled Hartree-Fock calculation $(\mathrm{KCH})$ in Table V). Even at the Lyman alpha line (1215.7 $\AA$ ) our results are still in reasonably good agreement with the semiempirical estimates. Figure 3 shows a plot of our

TABLE VII. Rayleigh scattering cross sections for heliumRPA. Comparison with other work.

\begin{tabular}{|c|c|c|c|}
\hline$\lambda(\AA)$ & $\begin{array}{l}\sigma(\omega)^{2} \\
\text { Experimental }^{\mathrm{b}}\end{array}$ & $\begin{array}{l}\sigma(\omega)^{2} \\
\text { This work }\end{array}$ & $\begin{array}{l}\sigma(\omega)^{2} \\
\text { Other theories }\end{array}$ \\
\hline 20587.0 & 0.003069 & 0.002789 & $\cdots$ \\
\hline 15300.0 & 0.01006 & 0.009151 & $\cdots$ \\
\hline 14756.0 & 0.01164 & 0.01058 & $\cdots$ \\
\hline 10142.0 & 0.05226 & 0.04750 & $\cdots$ \\
\hline 9227.0 & 0.07638 & 0.06941 & $0.0761^{d}$ \\
\hline 8266.8 & 0.1187 & 0.1079 & $0.118^{\mathrm{d}}$ \\
\hline 7247.2 & 0.2014 & 0.1830 & $0.201^{d}$ \\
\hline 5462.2 & 0.6284 & 0.5706 & $0.626^{\mathrm{d}}$ \\
\hline 4801.3 & 1.058 & 0.9600 & $1.054^{d}$ \\
\hline 4359.0 & 1.558 & 1.419 & $1.558^{d}$ \\
\hline 4047.0 & 2.105 & 1.917 & $2.104^{d}$ \\
\hline 3907.0 & 2.429 & 2.211 & $\cdots$ \\
\hline 3664.0 & 3.153 & 2.870 & $3.153^{d}$ \\
\hline 3342.0 & 4. 589 & 4.174 & $\cdots$ \\
\hline 3132.0 & 5.985 & 5.442 & $5.980^{d}$ \\
\hline 3022.0 & 6.929 & 6.300 & $\cdots$ \\
\hline 2968.0 & 7.462 & 6.783 & $\cdots$ \\
\hline 2926.0 & 7.913 & 7.192 & $\cdots$ \\
\hline 2894.0 & 8.280 & 7.524 & $\cdots$ \\
\hline 2753.0 & 10.17 & 9.240 & $10.17^{\mathrm{d}}$ \\
\hline 2500.0 & $\cdots$ & 13.76 & $13.74^{\bullet}(15)^{1}$ \\
\hline 2000.0 & $\cdots$ & 35.03 & $34.98^{\bullet}(138)^{t}$ \\
\hline 1500 & $\cdots$ & 121.7 & $121.5^{\bullet}(133)^{p}$ \\
\hline 1216 & $\cdots$ & 318.3 & $317.9^{\bullet}(353)^{p}$ \\
\hline 1000 & $\cdots$ & $8 \dot{4} 1.7$ & $840.7^{\bullet}(951)^{f}$ \\
\hline 800 & $\cdots$ & 3099 & $3096^{\bullet}(3590)^{t}$ \\
\hline 700 & $\cdots$ & 8654 & $8650^{\bullet}(10700)^{f}$ \\
\hline
\end{tabular}

In units of $10^{-28} \mathrm{~cm}^{2}$.

${ }^{b}$ Calculated from experimental refractivity data (which provides the necessary polarizability values).

${ }^{\prime}(12 S / 8 P)$ basis see text for details.

See Ref. 6 of text.

M. J. Jamieson, in Quantum Mechanics, the First Fifty Years, edited by W. C. Price, S. S. Chissick, and T. Ravensdale (Wiley, New York, 1973).

${ }^{\text {I}}$ Y. M. Chan and A. Dalgarno, Proc. R. Soc. Lond. 85, 227 (1965).

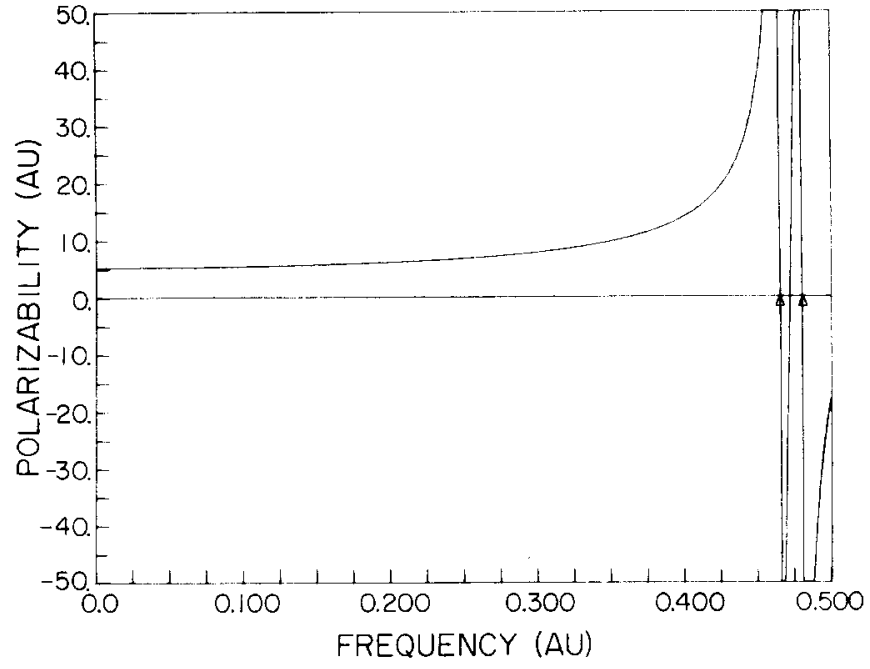

FIG. 5. Dispersion of $\alpha(\omega)$ for $\mathrm{H}_{2}(R=1.4$ a. u. $)$.

results (solid curve), the measured Verdet coefficients, and the moment theory bound results of Ref. 6 .

We also compute the Rayleigh scattering cross sections for helium using Eq. (11). These are listed in Table VI for the different basis sets used. It is clear that the $(12 S / 8 P)$ was complete enough to describe the property. In the case of the Rayleigh scattering cross section there are no direct experimental measurements. For this reason we compute the cross sections from the experimental refractivity data and compare them with our values in Table VII. Agreement is good over a wide range of frequencies, being better at lower values. We also compare it with other theoretical estimates available. Agreement is also good. For $\lambda \leqslant 2500 \AA$, the values listed in the last column of the table come from a timedependent Hartree-Fock calculation. Our results agree very closely with these as they should. Figure 4 shows

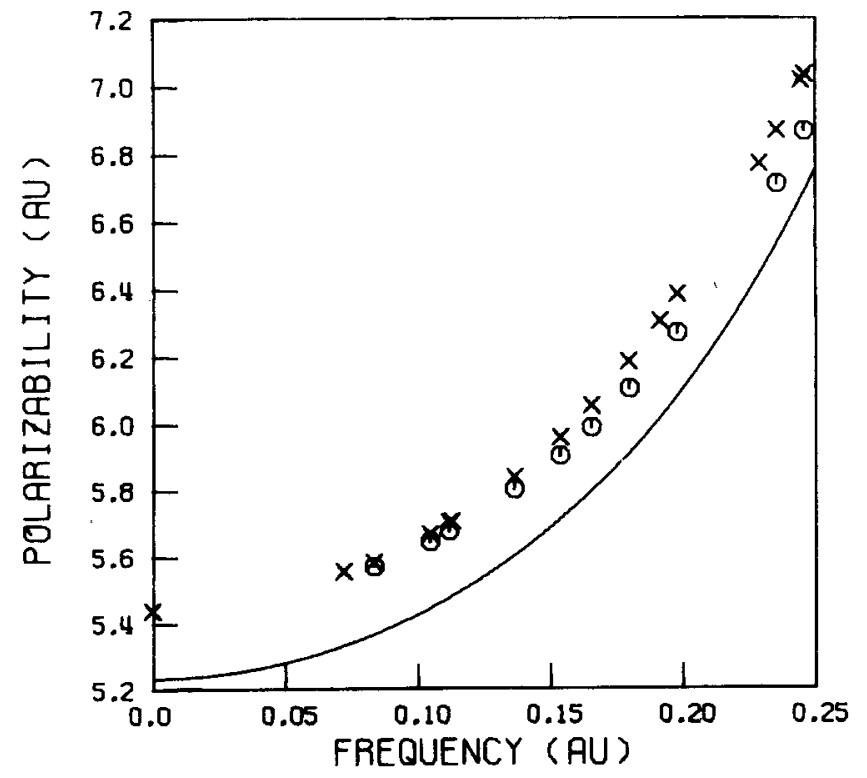

FIG. 6. Frequency-dependent polarizability of $\mathrm{H}_{2}(R=1.4$ a. u. $)$. Comparison of this work with experiment $(x)$ and results of Ref. $6(0)$. 
a plot of our results and "experimental" (in the sense explained above) data.

\section{Molecular hydrogen}

In Table VIII we list the results for the frequency-dependent polarizability of $\mathrm{H}_{2}$ and compare them with the experimental values and with the values derived from a semiempirical spectrum by Dalgarno and Victor. ${ }^{10}$ One must be careful in comparing these results with experimental or semiempirical results because they pertain to a fixed internuclear distance $(R=1.4 \mathrm{a} . \mathrm{u}$. in the present case of $\mathrm{H}_{2}$ ) and it has been shown recently ${ }^{11,12}$ that vibrational and rotational averaging can have a significant effect on the calculated properties (in some case of the order of $42 \%$ ). The anisotropy for instance is particularly sensitive to the averaging mainly at higher frequencies. Except for the results of Ford and Browne, ${ }^{12}$ no other theoretical work has included vibrational and rotational averaging effects. The values quoted from Ford and Browne's work in the table do not include averaging. Agreement with both the experimental and semiempirical data is in general very good even at

TABLE VIII. Frequency-dependent polarizability for $\mathrm{H}_{2}(R$ $=1.4 \mathrm{a}$. u. $)$. Comparison with experimental and other theories. Full polarizability $\alpha(\omega)$ in atomic units.

\begin{tabular}{|c|c|c|c|c|c|}
\hline$\lambda(\AA)$ & $\begin{array}{l}\text { Experi- } \\
\text { mental }\end{array}$ & $\begin{array}{l}\text { Model } \\
\text { spectrumb }\end{array}$ & $\begin{array}{l}\text { This } \\
\text { work }\end{array}$ & $\mathrm{FB}^{\mathrm{c}}$ & $P W L^{d}$ \\
\hline$\infty$ & 5.437 & 5.450 & 5.235 & 5.18 & 5.437 \\
\hline 6328.0 & 5. 554 & 5.554 & 5.331 & 5.28 & $\cdots$ \\
\hline 5462.3 & 5.582 & 5.591 & 5.365 & 5.31 & 5.588 \\
\hline 4556.0 & $\cdots$ & $5.655^{\circ}$ & 5.424 & $\cdots$ & $\cdots$ \\
\hline 4359.6 & 5.667 & 5.675 & 5.443 & 5.39 & 5.668 \\
\hline 4079.0 & 5.701 & 5.708 & 5.474 & $\cdots$ & 5.704 \\
\hline 3342.4 & 5.840 & 5.845 & 5.600 & $\cdots$ & 5.832 \\
\hline 3037.3 & $\cdots$ & $5.937^{\circ}$ & 5.685 & $\cdots$ & $\cdots$ \\
\hline 2968.1 & 5.960 & 5.963 & 5.708 & 5.65 & 5.948 \\
\hline 2753.6 & 6.055 & 6.056 & 5.794 & $\cdots$ & 6.036 \\
\hline 2379.1 & 6.303 & 6.299 & 6.017 & $\cdots$ & $\cdots$ \\
\hline 2302.9 & 6.384 & 6.368 & 6.079 & 6.01 & 6.332 \\
\hline 2278.0 & $\cdots$ & $6.392^{\circ}$ & 6.101 & $\cdots$ & $\cdots$ \\
\hline 1935.8 & 6.868 & 6.865 & 6.530 & $\cdots$ & 6.799 \\
\hline 1854.6 & 7.035 & 7.035 & 6.683 & 6.59 & 6.959 \\
\hline 1822.4 & $\cdots$ & $7.112^{\circ}$ & 6.752 & $\cdots$ & . \\
\hline 1700.0 & $\cdots$ & 7.471 & 7.071 & $\cdots$ & $\cdots$ \\
\hline 1600.0 & $\cdots$ & 7.872 & 7.424 & 7.31 & $\cdots$ \\
\hline 1500.0 & $\cdots$ & 8.431 & 7.908 & $\cdots$ & $\cdots$ \\
\hline 1400.0 & $\cdots$ & 9.262 & 8.610 & 8.44 & $\cdots$ \\
\hline 1300.0 & $\cdots$ & 10.62 & 9.714 & $\cdots$ & $\cdots$ \\
\hline 1215.7 & 12.8 & 12.76 & 11.29 & 11.02 & $\cdots$ \\
\hline
\end{tabular}

"From refractivity data: (a) J. Kock, Ark. Math. Astron. Fysik 8, 20 (1912) and M. Kirn, Ann. Physik 64, 566 (1912); (2) $\lambda=\infty \AA$, H. Schuler and K. L. Wolf, Z. Physik 34, 343 (1925); (3) $\lambda=6328 \AA$, N. J. Bridge and A. D. Buckingham, J. Chem. Phys. 40, 2733 (1964). Proc. R. Soc. A 295, 334 (1966); (4) $\lambda=1215.7 \AA$ (Lyman $\alpha$ ), P. Gill and D. W. O. Heddle, J. Opt. Soc. Am. 53, 848 (1963).

bodel semiempirical spectrum of $\mathrm{H}_{2}$ constructed by G. A. Victor and A. Dalgarno, J. Chem. Phys. 50, 2535 (1969). 'Direct sum-over-states calculation of Ref. 12 of text.

${ }^{\text {d}}$ Padé approximants calculation of P. W. Langhoff and M. Karplus, Ref. 7.

'Calculated from the model spectrum of G. A. Victor and A. Dalgarno, J. Chem. Phys. 50, 2535 (1969).
TABLE IX. Frequency dependent anisotropy for $\mathrm{H}_{2}(R=1.4$ a. u. $)$. Comparison with other theories $[\gamma(\omega)$ given in atomic units].

\begin{tabular}{llccc}
\hline \hline & $\begin{array}{l}\text { Model } \\
\text { Spectrum }\end{array}$ & $\begin{array}{l}\text { This } \\
\text { work }\end{array}$ & FB $^{\text {b }}$ & PWL $^{\mathrm{c}}$ \\
\hline$\infty$ & 2.029 & 1.865 & 1.84 & 1.826 \\
6328.0 & 2.092 & 1.919 & 1.90 & $\ldots$ \\
5462.3 & 2.115 & 1.938 & 1.92 & $\ldots$ \\
4556.0 & 2.155 & 1.972 & $\ldots$ & 1.930 \\
4359.6 & 2.168 & 1.982 & 1.96 & $\ldots$ \\
4079.0 & 2.188 & 2.000 & $\ldots$ & $\ldots$ \\
3342.4 & 2.274 & 2.073 & $\ldots$ & $\ldots$ \\
3037.3 & 2.333 & 2.122 & $\ldots$ & 2.074 \\
2968.1 & 2.350 & 2.136 & 2.11 & $\ldots$ \\
2753.6 & 2.410 & 2.186 & $\ldots$ & $\ldots$ \\
2379.1 & 2.569 & 2.319 & $\ldots$ & $\ldots$ \\
2302.9 & 2.615 & 2.357 & 2.32 & $\ldots$ \\
2278.0 & 2.631 & 2.370 & $\ldots$ & 2.311 \\
1935.8 & 2.958 & 2.637 & $\ldots$ & $\ldots$ \\
1854.6 & 3.080 & 2.734 & 2.69 & $\ldots$ \\
1822.4 & 3.136 & 2.779 & $\ldots$ & 2.703 \\
1700.0 & 3.401 & 2.988 & $\ldots$ & $\ldots$ \\
1600.0 & 3.710 & 3.226 & 3.16 & $\ldots$ \\
1500.0 & 4.159 & 3.564 & $\ldots$. & $\ldots$ \\
1400.0 & 4.871 & 4.077 & 3.95 & $\ldots$ \\
1300.0 & 6.158 & 4.939 & $\ldots$. & $\ldots$ \\
1215.7 & 8.495 & 6.285 & 6.12 & $\ldots$ \\
\hline \hline
\end{tabular}

${ }^{2}$ Model semiempirical spectrum of $\mathrm{H}_{2}$ constructed by $\mathrm{G}$. A. Victor and A. Dalgarno, J. Chem. Phys. 50, 2535 (1969). Direct sum-over-states calculation of Ref. 12 of text. Unaveraged results shown for comparison. See text.

${ }^{\mathrm{C}}$ Moment theory bounds results of Ref. 6 of text.

higher $\omega$ values where the averaging effects should be greater. Our agreement with other theories is excellent (including the accurate Ford and Browne results). Figure 5 shows our dispersion curve for the total polarizability out to the second pole. Figure 6 shows a more detailed view (expanded scale) of this curve together with experimental points.

Table IX shows our computed anisotropies. In this case there are practically no measurements at all (ex-

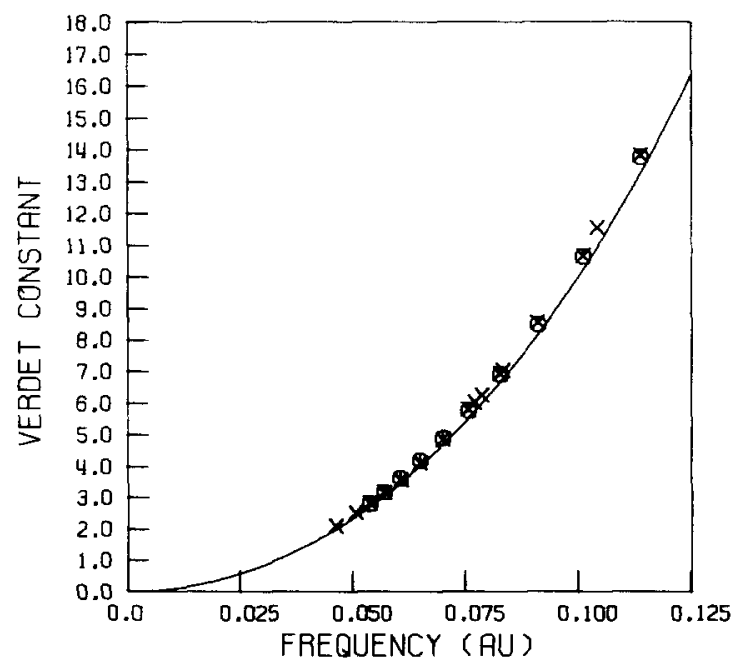

FIG. 7. Verdet constants of $\mathrm{H}_{2}(R=1.4$ a. u. $)$. Comparison of this work with experiment $(x)$ and results of Ref. 6 (o) (units are microminutes. Oersted ${ }^{-1} \cdot \mathrm{cm}^{-1}$ at $0^{\circ} \mathrm{C}$ and $\left.1 \mathrm{~atm}\right)$. 
TABLE X. Verdet constants $V(\omega)^{2}$ of $\mathrm{H}_{2}(R=1.4$ a. u. $)$.

\begin{tabular}{lcccc}
\hline \hline$\lambda(\AA)$ & $\begin{array}{l}\text { Experi- } \\
\text { ment }^{\mathrm{b}}\end{array}$ & $\begin{array}{l}\text { Model } \\
\text { spectrum }\end{array}$ & $\begin{array}{l}\text { This } \\
\text { work }\end{array}$ & PWL $^{\text {d }}$ \\
\hline$\infty$ & $\cdots$ & 0.0 & 0.0 & $\ldots$ \\
9875 & 2.11 & 2.15 & 1.99 & $\ldots$ \\
9000 & 2.53 & 2.59 & 2.41 & $\ldots$ \\
8500 & 2.83 & 2.91 & 2.71 & 2.81 \\
8000 & 3.18 & 3.30 & 3.07 & 3.18 \\
7500 & 3.59 & 3.77 & 3.50 & 3.64 \\
7000 & 4.14 & 4.35 & 4.04 & 4.19 \\
6500 & 4.88 & 5.07 & 4.71 & 4.89 \\
6000 & 5.81 & 5.99 & 5.56 & 5.78 \\
5893 & 6.03 & 6.22 & 5.77 & $\ldots$ \\
5780 & 6.27 & 6.48 & 6.01 & $\ldots$ \\
5500 & 6.94 & 7.20 & 6.68 & 6.93 \\
5460 & 7.06 & 7.31 & 6.78 & $\cdots$ \\
5000 & 8.55 & 8.82 & 8.17 & 8.49 \\
4500 & 10.7 & 11.1 & 10.2 & 10.65 \\
4360 & 11.4 & 11.9 & 11.0 & $\cdots$ \\
4000 & 13.8 & 14.3 & 13.3 & 13.78 \\
3635 & 17.2 & 17.8 & 16.4 & $\ldots$ \\
3342.4 & $\ldots$. & 21.6 & 19.9 & $\ldots$ \\
2500 & $\cdots$ & 44.3 & 40.5 & $\ldots$ \\
2000 & $\ldots$ & 84.1 & 76.0 & $\cdots$ \\
\hline \hline
\end{tabular}

2In units of microminutes. Oersted ${ }^{-1} \cdot \mathrm{cm}^{-1}$ (at $0^{\circ} \mathrm{C}$ and $1 \mathrm{~atm}$ ). ${ }^{\text {Beference } 29 .}$

${ }^{\mathrm{C}}$ Model semiempirical spectrum of $\mathrm{H}_{2}$ constructed by $\mathrm{G}$. A. Victor and A. Dalgarno, J. Chem. Phys. 50, 2535 (1969). With the exception of the points at $\lambda=\infty \AA$ and $\lambda=3342.4 \AA$ the values were calculated from their spectrum.

Moment theory bounds results of Ref. 6 .

cept at $6328 \AA$-see table) so the semiempirical spectrum of Dalgarno and Victor ${ }^{10}$ is used as a reliable comparison guide. The difference between our values of the anisotropy at higher frequencies and those from the model spectrum are within the possible averaging effects found by Ford and Browne. ${ }^{12}$ In fact our result agrees very well with their unaveraged result.

Table X compares our calculated Verdet constants with experimental and semiempirical data as well as with other theoretical results. Here the limitation of
TABLE XI. Rayleigh scattering cross sections for $\mathrm{H}_{2} \quad(R=1.4$ a.u.) $\sigma(\omega) .^{a}$

\begin{tabular}{lccc}
\hline \hline$\lambda(\AA)$ & Model spectrum $^{\mathrm{b}}$ & This work & PWL $^{\mathbf{c}}$ \\
\hline$\infty$ & 0.0 & 0.0 & $\ldots$ \\
6328.0 & 0.0569 & 0.0523 & $\ldots$ \\
5462.3 & 0.104 & 0.0953 & 0.1 \\
4359.6 & 0.264 & 0.242 & 0.264 \\
4079.0 & 0.348 & 0.319 & 0.348 \\
3342.4 & 0.811 & 0.742 & 0.809 \\
2968.1 & 1.36 & 1.24 & 1.35 \\
2753.6 & 1.89 & 1.73 & 1.88 \\
2379.1 & 3.68 & 3.35 & $\cdots$ \\
2302.9 & 4.29 & 3.89 & 4.24 \\
1935.8 & 10.0 & 9.02 & 9.82 \\
1854.6 & 12.5 & 11.2 & 12.2 \\
1700.0 & 20.0 & 17.8 & $\cdots$ \\
1600.0 & 28.4 & 25.1 & $\ldots$ \\
1500.0 & 42.4 & 37.0 & $\cdots$ \\
1400.0 & 68.0 & 58.1 & $\ldots$ \\
1300.0 & 122 & 100 & $\ldots$ \\
1215.7 & 235 & 179 & $\cdots$ \\
\hline \hline
\end{tabular}

IIn units of $10^{-26} \mathrm{~cm}^{2}$.

${ }^{b}$ Model semiempirical spectrum of $\mathrm{H}_{2}$ constructed by G. A. Victor and A. Dalgarno, J. Chem. Phys. 50, 2535 (1969). ${ }^{c}$ Moment theory bounds results of Ref. 6 .

the Becquerel formula (on which our results are based) and the lack of rotational and vibrational averaging should again be kept in mind. Our results are in good agreement with the experimental values $(\leqslant 5 \%$ in most cases). At much higher frequencies, for which experiments are unavailable, our results compare well with the semiempirical estimates $(\sim 10 \%)$. Figure 7 shows a plot of our results and the experimental Verdet data. We have also computed Rayleigh scattering cross sections using our $\alpha(\nu)$ and $\gamma(\omega)$ data in Eq. (11). The results are shown in Table XI. Since no direct measurements are available we compare with the model spectrum as before. The same remarks about the averaging made before apply here again. At lower frequencies our results are within about $10 \%$ of the semiempirical

TABLE XI. Sum rules $S(k)$ for helium and molecular hydrogen.

\begin{tabular}{|c|c|c|c|c|c|c|c|}
\hline System & $S(2)$ & $S(1)$ & $S(0)$ & $S(-1)$ & $S(-2)$ & $S(-4)$ & $S(-6)$ \\
\hline $\mathrm{He}$ & $\begin{array}{l}30.37^{\mathrm{a}} \\
(30.3325)^{\mathrm{b}}\end{array}$ & $\begin{array}{c}4.126^{\mathrm{a}} \\
(4.0837)^{\mathrm{b}}\end{array}$ & $\begin{array}{l}2.001 \\
(2)^{\mathrm{c}}\end{array}$ & $\begin{array}{c}1.478 \\
(1.505)^{\mathrm{b}}\end{array}$ & $\begin{array}{l}1.322 \\
(1.3838)^{d}\end{array}$ & $\begin{array}{l}1.386 \\
(1.550)^{d}\end{array}$ & $\begin{array}{l}1.730 \\
(2.066)^{\mathrm{d}}\end{array}$ \\
\hline $\mathrm{H}_{2}$ & $\begin{array}{l}2.447 \\
(3.6929)^{\bullet}\end{array}$ & $\begin{array}{l}1.666 \\
(1.7035)^{\circ}\end{array}$ & $\begin{array}{l}1.999 \\
(2)^{\mathrm{c}}\end{array}$ & $\begin{array}{l}3.053 \\
(3.1671)^{8}\end{array}$ & $\begin{array}{l}5.234 \\
(5.439)^{d}\end{array}$ & $\begin{array}{l}18.25 \\
(20.02)^{d}\end{array}$ & $\begin{array}{c}71,29 \\
(81.61)^{d}\end{array}$ \\
\hline $\mathbf{H}_{2}^{\prime \prime}$ & $\begin{array}{c}1.639 \\
(0.82)^{\mathrm{g}}\end{array}$ & $\begin{array}{c}1.330 \\
(1.214)^{\mathrm{g}}\end{array}$ & $\begin{array}{l}1.994 \\
(2)^{c}\end{array}$ & $\begin{array}{l}3.478 \\
(3.4452)^{\mathrm{h}}\end{array}$ & $\begin{array}{l}6.478 \\
(6.3517)^{\mathrm{h}}\end{array}$ & $\begin{array}{c}25.03 \\
(27.55)^{\mathbf{g}}\end{array}$ & 104.5 \\
\hline $\mathbf{H}_{2}^{1}$ & $\begin{array}{c}2.851 \\
(1.93)^{\mathrm{g}}\end{array}$ & $\begin{array}{c}1.835 \\
(1.674)^{\mathrm{B}}\end{array}$ & $\begin{array}{l}2.002 \\
(2)^{\mathrm{c}}\end{array}$ & $\begin{array}{l}2.841 \\
(2.8264)^{\bullet}\end{array}$ & $\begin{array}{c}4.613 \\
(4.750)^{\circ}\end{array}$ & $\begin{array}{c}14.86 \\
(15.67)^{\mathrm{g}}\end{array}$ & 54.66 \\
\hline
\end{tabular}

${ }^{2}$ These values pertain to the (10S/13P) calculation on He (see text for details).

${ }^{b} \mathrm{C}$. L. Pekeris, Phys. Rev. 112, 1649 (1958).

${ }^{\mathrm{C}}$ Exact value.

dP. W. Langhoff and M. Karplus, J. Opt. Soc. Am. 59, 863 (1969).

'W. Kolos and L. Wolniewicz, J. Chem. Phys. 41, 3663 (1964); 46, 1426 (1967).

${ }^{2}$ R. Kamikawai, T. Watanabe, and A. Amemiya, Phys. Rev. 184, 303 (1969).

G. A. Victor and A. Dalgarno, J. Chem. Phys. 50, 2535 (1969).

Reference 12 . 
TABLE XIII. van der Waals force coefficients for He and $\mathrm{H}_{2}$ interactions. ${ }^{2}$

\begin{tabular}{llllllll}
\hline Interaction & $\mathrm{He}-\mathrm{He}$ & $\mathrm{He}-\mathrm{H}_{2}{ }^{\mathrm{b}}$ & $\mathrm{He}-\mathrm{H}_{2}(\|)$ & $\mathrm{He}-\mathrm{H}_{2}(\mathrm{H})$ & $\mathrm{H}_{2}(\|)-\mathrm{H}_{2}(\|)$ & $\mathrm{H}_{2}(\perp)-\mathrm{H}_{2}(\|)$ & $\mathrm{H}_{2}(\perp)-\mathrm{H}_{2}(1)$ \\
\hline This work & 1.374 & 3.796 & 0.748 & 0.575 & 2.749 & 2.069 & 1.563 \\
Other work & $1.375^{\mathrm{c}}$ & $3.794^{\mathrm{d}}$ & $0.746^{\mathrm{c}}$ & $0.5755^{\mathrm{c}}$ & $2.732^{\mathrm{c}}$ & $2.063^{\mathrm{c}}$ & $1.564^{\mathrm{c}}$ \\
\hline \hline
\end{tabular}

Entries refer to the $C_{a b}$ 's defined in the text [Eq. (2)].

${ }^{b}$ Full polarizability used for $\mathrm{H}_{2}$.

'P. W. Langhoff, R. G. Gordon, and M. Karplus, J. Chem. Phys. 55, 2126 (1971).

${ }^{d}$ Computed from data in Ref. c according to Eq. (6a) of that work.

estimates deviating more strongly at higher frequencies. This is due mainly to the lack of averaging in our anisotropy value. A plot of our results and the semiempirical values is given in Fig. 8.

\section{B. Sum rules and van der Waals coefficients}

The Thomas-Reiche-Kuhn sum rule (which the exact RPA results satisfies identically) is just one of a set of generalized energy weighted sum rules $S(k)$ defined by

$$
S(k)=\sum_{n} f_{n o}\left(E_{n}-E_{o}\right)^{k}=\sum_{n} f_{n o} \omega_{n o}^{k} .
$$

For diatomic molecules this is usually broken up into two components $S^{\prime \prime}(k)$ and $S^{\perp}(k)$ involving transitions polarized either parallel or perpendicular to the molecular axis. With these definitions we have

$$
\begin{aligned}
& S^{\prime \prime}(k)=3 \sum_{n} f_{n o}^{\prime \prime} \omega_{n o}^{k}, \\
& S^{1}(k)=\frac{3}{2} \sum_{n} f_{n o}^{1} \omega_{n o}^{k},
\end{aligned}
$$

and

$$
S(k)=\frac{1}{3}\left[2 S^{\perp}(k)+S^{\prime \prime}(k)\right] .
$$

Thus the Thomas-Reiche-Kuhn sum rule $S(0)$ can be

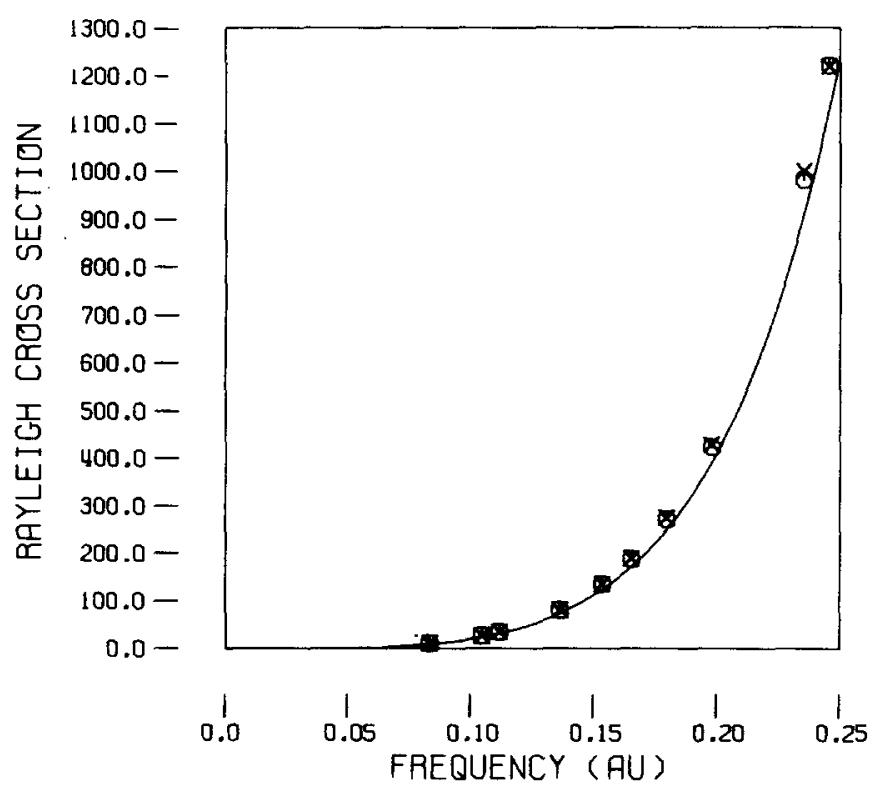

FIG. 8. Rayleigh scattering cross sections for $\mathrm{H}_{2}(R=1.4$ a. u. ). Comparison of this work with semiempirical estimates and results of Ref. 6 ( $*$ and $\circ$, respectively, in the figure). Units are $10^{-28} \mathrm{~cm}^{2}$. written for diatomic molecules as

$$
S^{\prime \prime}(0)=S^{1}(0)=S(0)=N \text {, }
$$

$N$ being the number of electrons in the system.

We have used our oscillator strength distributions for $\mathrm{He}$ and $\mathrm{H}_{2}$ to compute several of these sums and have collected them in Table XII where they are compared with previous estimates. Good agreement is found for a large number of these sum rules. This explains in part the success of RPA theory in predicting secondorder optical properties.

We have also calculated several dipole interaction coefficients through direct use of Eq. (2) and a direct sum over our discrete spectrum. These results in Table XIII agree well with the best previous estimates from Pade approximants and Gaussian quadrature procedures. ${ }^{8}$

\section{CONCLUSIONS}

A sum-over-states procedure using a spectrum (excitation energies and oscillator strengths) from the random phase approximation in a completely square-integrable (Gaussian) basis is shown to be accurate and practical for the calculation of dipole dynamic polarizabilities and anisotropies. Applications are made to helium and molecular hydrogen. It is shown that essentially exact solutions of the RPA equations (even beyond the first pole) can be obtained using only boundlike (Gaussian) basis functions on which no special constraints are imposed (e.g., sum rule constraints). This is important since second-order optical properties formally involve the continuum.

Our dynamic polarizability results for helium are in good agreement with the exact numerical solution provided by Alexander and Gordon. ${ }^{26}$ The results for molecular hydrogen also agree well with the best calculations available.

The RPA satisfies the Thomas-Reiche-Kuhn sum rule exactly, and we have found that it also satisfies approximately a large number of other related energy weighted sum rules.

The accuracy of the results and the practicality and applicability of the procedure to larger molecular systems $\left(\mathrm{CO}, \mathrm{N}_{2}, \mathrm{CO}_{2}, \mathrm{H}_{2} \mathrm{O}, \mathrm{C}_{6} \mathrm{H}_{6} \text {, etc. }\right)^{22}$ shows that the proposed method can be of wide use in the study of second-order properties and dispersion force coefficients.

\section{ACKNOWLEDGMENTS}

One of us (P.H.S.M.) thanks Marco A. C. Nascimento 
for his interest and helpful discussions. He thanks Anna Martin for invaluable help in the preparation of the manuscript. He also acknowledges the CNPq (Brazil) for financial support and the Physical Chemistry Department of the Universidade Federal do Rio de Janeiro for granting him a leave of absence to carry out his graduate work at Caltech.

\section{APPENDIX A}

Long range dispersion force coefficients used in Eq. (2). A stands for atom and $M$ for molecule, 1 for the perpendicular component and $\|$ for the parallel component of the molecular polarizability.

$\begin{array}{ll}\text { Interaction } & \mathfrak{e}(a, b) \\ \mathrm{A}-\mathrm{A} & 3 / 2 \\ \mathrm{~A}-\mathrm{M}(\perp+\|) & 3 / 2 \\ \mathrm{~A}-\mathrm{M}(\mathrm{II}) & 3 / 4 \\ \mathrm{~A}-\mathrm{M}(\perp) & 3 / 8 \\ \mathrm{M}(1)-\mathrm{M}(\|) & 9 / 4 \\ \mathrm{M}(\|)-\mathrm{M}(1) & 9 / 8 \\ \mathrm{M}(\perp)-\mathrm{M}(\perp) & 9 / 16\end{array}$

\section{APPENDIX B: BASIS SETS USED IN CALCULATIONS}

Helium was calculated in three Gaussian bases: $(11 S / 5 P)$, $(12 S / 8 P)$, and $(10 S / 13 P)$. The $(11 S / 5 P)$ basis is as follows :

\section{S functions}

\begin{tabular}{|c|c|}
\hline Exponent & Coefficient \\
\hline 3292.694000 & 0.0046146 \\
\hline 488.894100 & 0.0365754 \\
\hline 108.772300 & 0.1978343 \\
\hline 30.179900 & 0.8270723 \\
\hline 9.789053 & 1. \\
\hline 3.522261 & 1. \\
\hline 1.352436 & 1. \\
\hline 0.552610 & 1. \\
\hline 0.240920 & 1. \\
\hline 0.107951 & 1. \\
\hline 0.048370 & 1. \\
\hline 0.021674 & 1. \\
\hline 0.009712 & 1. \\
\hline 0.003000 & 1. \\
\hline \multicolumn{2}{|c|}{ P functions } \\
\hline 1.553506 & 0.0710316 \\
\hline 0.368859 & -1.037338 \\
\hline 0.119213 & 1.0 \\
\hline 0.044914 & 1.0 \\
\hline 0.018133 & 1.0 \\
\hline 0.0073207 & 1.0 \\
\hline
\end{tabular}

The $(12 S / 8 P)$ basis has the same $S$ functions as the $(11 S / 5 P)$ above except for an additional diffuse one with exponent 0.001 . The $P$ part of the basis consists of eight $P$ functions with exponents $4.5,1.45337,0.34627$, $0.11191,0.04216,0.01702,0.01127$, and 0.0051 . the $(11 S / 5 P)$ but with the function with exponent of 0.003 deleted. The exponents for the $P$ functions are 121.5 , $40.55,13.5,4.5,1.458369,1.00,0.34627,0.111912$, $0.042163,0.0170230,0.0112650,0.0051045$, and 0.0017 .

Molecular hydrogen was calculated in an uncontracted $(8 S / 5 P)$ basis taken mostly from Huzinaga's basis. ${ }^{24}$ Only another diffuse $S$ function was added to the $(7 S / 5 P)$ basis of Huzinaga. ${ }^{24}$ Also a $d_{z z}, d_{z x}$, and $d_{z y}$ function each with exponent 0.35 was placed on each hydrogen atom. On the center of the molecule an additional $S$ function (exponent 0.0065 ) and a $P$ function (exponent $0.0065)$ were included. The $(8 S / 5 P)$ part of the basis is

\section{S functions}

$\begin{array}{cc}\text { Exponent } & \text { Coefficient } \\ 213.5134 & 1.0 \\ 31.93095 & 1.0 \\ 7.15706 & 1.0 \\ 1.97352 & 1.0 \\ 0.62879 & 1.0 \\ 0.22444 & 1.0 \\ 0.087463 & 1.0 \\ 0.038484 & 1.0\end{array}$

$P$ fronctions

$\begin{array}{ll}2.10005 & 1.0 \\ 0.498629 & 1.0 \\ 0.161153 & 1.0 \\ 0.060715 & 1.0 \\ 0.024513 & 1.0\end{array}$

\section{APPENDIX C: RPA EXCITATION SPECTRA}

Excitation energies, $\omega_{o n}$, and oscillator strengths, $f_{\text {on }}$, used in the calculations.

Helium $(11 S / 5 P)$

$\omega_{\text {or }}(\mathrm{eV})$

$f_{\text {on }}$

21.69318

0.2466

23.50479

0.0711

24.71259

0.1362

29.21289

0.4380

46.7849

0.7269

$\sum f_{\text {on }}=1.6188$

Helium $(12 S / 8 P)$

$\omega_{\text {on }}(\mathrm{eV})$

$f_{\text {on }}$

21.68579

0.2520

23.49869

0.0705

24.24010

0.0528

25.49229

0.1338

29.55789

0.3938

45.19099

0.7080

114.65388

0.3696

373.37329

The $(10 S / 13 P)$ basis has the same $S$ components as 
Helium $(10 S / 13 P)$

$\begin{array}{cl}\omega_{\text {on }}(\mathrm{eV}) & f_{\text {on }} \\ 21.68559 & 0.2520 \\ 23.49860 & 0.0705 \\ 24.14690 & 0.0288 \\ 24.51109 & 0.0372 \\ 25.65469 & 0.1290 \\ 29.63460 & 0.3834 \\ 44.30089 & 0.6525 \\ 88.73340 & 0.3372 \\ 188.7334 & 0.0873 \\ 443.1355 & 0.0207 \\ 1217.095 & 0.0018 \\ 3672.933 & 0.0006 \\ & \sum f_{\text {on }}=2.001\end{array}$

Molecular hydrogen

\begin{tabular}{|c|c|}
\hline \multicolumn{2}{|c|}{$\mathrm{H}_{2}{ }^{1} \Pi_{v}$ states } \\
\hline$\omega_{o n}(\mathrm{eV})$ & $f_{o n}[$ See Eq. (3).] \\
\hline 13.08204 & 0.329600 \\
\hline 14.75701 & 0.082748 \\
\hline 16.40558 & 0.259426 \\
\hline 23.26047 & 0.423587 \\
\hline 45.29939 & 0.089050 \\
\hline 47.73329 & 0.128098 \\
\hline 160.01324 & 0.021847 \\
\hline & $\sum f_{o n}=2.0017$ \\
\hline \multicolumn{2}{|c|}{$\mathrm{H}_{2}{ }^{1} \Sigma_{u}^{+}$States } \\
\hline$\omega_{o n}(e V)$ & $f_{o n}$ [See Eq. (4).] \\
\hline 12.66065 & 0.293237 \\
\hline 14.59307 & 0.062034 \\
\hline 15.67013 & 0.076577 \\
\hline 17.54407 & 0.042900 \\
\hline 20.07199 & 0.085979 \\
\hline 25.11337 & 0.066522 \\
\hline 33.91255 & 0.026622 \\
\hline 50.64389 & 0.002734 \\
\hline 55.53005 & 0.003803 \\
\hline 94.04312 & $6.33256 \times 10^{-4}$ \\
\hline 152.07542 & $4.43154 \times 10^{-5}$ \\
\hline 218.02448 & $3.50420 \times 10^{-3}$ \\
\hline 482.13574 & $1.14551 \times 10^{-5}$ \\
\hline \multirow[t]{2}{*}{1913.02540} & $2.87545 \times 10^{-6}$ \\
\hline & $\sum f_{o n}=1.994$ \\
\hline
\end{tabular}

*Supported by a grant from the National Science Foundation. tCNPq (Brasil) Predoctoral Fellow.

+⿳亠口冋t Contribution No. 4879 .

§Permanent address: Department of Chemistry, California Institute of Technology, Pasadena, CA 91109.

${ }^{1}$ S. A. Korff and G. Breit, Rev. Mod. Phys, 4, 471 (1932).

${ }^{2}$ R. Serber, Phys. Rev. 41, 489 (1932).

${ }^{3}$ I. L. Fablenski, Molecular Scattering of Light (Plenum, New York, 1968).

${ }^{4}$ H. B. G. Casimir and D. Polder, Phys. Rev. 73, 360 (1948); A. Dalgarno, Adv. Chem. Phys, 12, 143 (1967).

${ }^{5}$ The Raman Effect, edited by A. Anderson (Dekker, New York, 1971).

${ }^{6}$ P. W. Langhoff, J. Chem. Phys. 57, 2604 (1972).

${ }^{7} \mathrm{P}$. W. Langhoff and M. Karplus, J. Chem. Phys. 52, 1435 (1970).

${ }^{8}$ See P. W. Langhoff, R. G. Gordon, and M. Karplus, J. Chem. Phys. 55, 2126 (1971).

${ }^{9}$ See P. T. Gee and K. T. Tang, Phys. Rev. A 7, 1863 (1973) and the references therein. See also Ref. 8, Footnote 28.

${ }^{10}$ See for instance A. E. Kingston, J. Opt. Soc. A. 54, 1145 (1964); G. A. Victor and A. Dalgarno, J. Chem. Phys. 50, 2535 (1969).

${ }^{11}$ A. Dalgarno, A. L. Ford, and J. C. Browne, Phys. Rev. Lett. 27, 1033 (1971).

${ }^{12}$ A. L. Ford and J. C. Browne, Phys. Rev. A 7, 418 (1973).

${ }^{13}$ M. Karplus and H. J. Kolker, J. Chem. Phys. 39, 1413 (1963).

${ }^{14}$ M. Karplus and H. J. Kolker, J. Chem. Phys. 41, 3956 (1964).

${ }^{15}$ H. J. Kolker and H. H. Michels, J. Chem. Phys. 43, 1027 (1965).

${ }^{16}$ A. Dalgarno and S. T. Epstein, J. Chem. Phys. 50, 2837 (1969).

${ }^{17} \mathrm{~J}$. C. Salter, Quantum Theory of Atomic Structure (McGrawHill, New York, 1969), Vol. 1, p. 154.

${ }^{18}$ See for example J. O. Hirschfelder and W. J. Meath, Adv. Chem. Phys. 12, 3 (1967).

${ }^{19}$ A. Dalgarno and W. D. Davison, Adv. At. Mol. Phys. 2, 1 (1966); also A. Dalgarno, Adv. Chem. Phys. 12, 143 (1967). ${ }^{20}$ T. Shibuya and V. McKoy, Phys. Rev. A 2, 2208 (1970).

${ }^{21}$ T. Shibuya, J. Rose, and V. McKoy, J. Chem. Phys. 58, 500 (1973).

${ }^{22}$ T. Shibuya and V. McKoy, Phys. Rev. A 2, 2208 (1970); J. Rose, T. Shibuya, and V. McKoy, J. Chem. Phys. 58, 74 (1973); J. Rose, T. Shibuya, and V. McKoy, ibid. 60, 2700 (1974); D. L. Yeager and V. McKoy, ibid. 60, 2714 (1974).

${ }^{23}$ R. A. Harris, J. Chem. Phys. 50, 3947 (1969); see also D. J. Thouless, Nucl. Phys. 22, 781 (1961).

${ }^{24} \mathrm{~S}$. Huzinaga, J. Chem. Phys, 42, $1293(1965)$.

${ }^{25} \mathrm{C}$. W. McCurdy, Jr., and V. McKoy, J. Chem. Phys. 61, 2820, (1974).

${ }^{26}$ M. H. Alexander and R. G. Gordon, J. Chem. Phys. 56, 3823 (1972).

${ }^{27}$ V. G. Kaveeshwar, K. T. Chung, and R. P. Hurst, Phys. Rev. 172, 35 (1968).

${ }^{28}$ L. R. Ingersoll and D. H. Liebenberg, J. Opt. Soc. Am. 44, 566 (1954); 46, 538 (1956). 